\title{
ISS of interconnected impulsive systems with and without time-delays
}

\author{
Sergey Dashkovskiy ${ }^{*, 1}$ Michael Kosmykov ${ }^{*, 2}$ Lars Naujok $^{*, 1}$ \\ * University of Bremen, Centre of Industrial Mathematics, \\ P.O.Box 330440, 28334 Bremen, Germany
}

\begin{abstract}
We consider networks of impulsive systems with and without time-delays and investigate under which conditions the whole network is input-to-state stable (ISS). We provide conditions on the size of the time intervals between impulses and on the interconnection structure that guarantee ISS of the overall system, where Lyapunov and Lyapunov-Razumikhin functions for the subsystems are used. The condition on the interconnection allows to construct a Lyapunov (-Razumikhin) function and a corresponding gain for the whole system.
\end{abstract}

Keywords: Impulsive systems, input-to-state stability, interconnected systems, time-delay systems, Lyapunov methods, small-gain theorems

\section{INTRODUCTION}

Impulsive systems combine continuous and discontinuous behaviors of a dynamical system. The continuous dynamics is typically described by ordinary differential equations and the discontinuous behavior are instantaneous state jumps that occur at given time instants, also referred to as impulses. Impulsive systems are closely connected to hybrid systems and switched systems and have wide applications in network control, engineering, biological or economical systems, see van der Schaft and Schumacher [2000], Haddad et al. [2006], Shorten et al. [2007].

In this paper we study the input-to-state stability (ISS) property of impulsive systems with external inputs. ISS was first introduced for continuous systems in Sontag [1989]. A useful tool to verify the ISS property for continuous systems are Lyapunov functions (see Sontag and Wang [1995]) as well as for other variants of ISS, namely input-to-state dynamical stability (ISDS) (Grüne [2002]), local ISS (LISS) (Sontag and Wang [1996]) and integral-ISS (iISS) (Sontag [1998]). Investigations of ISS for hybrid systems can be found in Cai and Teel [2009]. For time-delay systems the ISS property can be verified by Lyapunov-Razumikhin functions (Teel [1998]) or Lyapunov-Krasovskii functionals (Pepe and Jiang [2006]).

For impulsive systems the ISS and iISS properties were studied in Hespanha et al. [2008] for the delay-free case and in Chen and Zheng [2009] for time-delay systems. By the help of exponential ISS Lyapunov(-Razumikhin) functions and an (reverse) average dwell-time (ADT) condition the ISS property for impulsive (time-delay) systems was proved.

\footnotetext{
1 Sergey Dashkovskiy and Lars Naujok are supported by the German Research Foundation (DFG) as part of the Collaborative Research Center 637 "Autonomous Cooperating Logistic Processes: A Paradigm Shift and its Limitations".

2 Michael Kosmykov is supported by the Volkswagen Foundation as part of the Project Nr. I/82684 "Dynamic Large-Scale Logistics Networks".
}

We are interested in the ISS property for interconnections of impulsive systems with and without time-delays. The first results on the ISS property for the delay-free case were given for two coupled continuous systems in Jiang et al. [1994] and for an arbitrarily large number $(n \in \mathbb{N})$ of coupled systems in Dashkovskiy et al. [2007], using a smallgain condition. Lyapunov versions of the ISS small-gain theorems were proved in Jiang et al. [1996] (two systems) and Dashkovskiy et al. [2009] ( $n$ systems), for the ISDS property in Dashkovskiy and Naujok [2010], for LISS in Dashkovskiy and Rüffer [2010] and for iISS in Ito [2006] (two systems) and Ito et al. [2009] ( $n$ systems), where Lyapunov functions for the overall system are constructed. ISS for interconnected hybrid systems was investigated in Nesic and Teel [2008] (two systems) and Dashkovskiy and Kosmykov [2009] ( $n$ systems). A general approach of the verification of the ISS property for interconnected systems can be found in Karafyllis and Jiang [2009].

In this paper we prove that under a small-gain condition and the (reverse) ADT condition for interconnected impulsive systems according to Hespanha et al. [2008] the overall system is again ISS and construct the exponential ISS Lyapunov(-Razumikhin) function and the corresponding gain of the whole system.

The paper is organized as follows: In Section 2 we note some basic definitions. The first main result, the ISS smallgain theorem for interconnected delay-free impulsive systems can be found in Section 3. Section 4 contains the second main result, the ISS small-gain theorem for interconnected impulsive systems with time-delays. Finally Section 5 concludes this paper with a short summary.

\section{PRELIMINARIES}

By $x^{T}$ we denote the transposition of a vector $x \in \mathbb{R}^{N}$, $N \in \mathbb{N}$, furthermore $\mathbb{R}_{+}:=[0, \infty)$ and $\mathbb{R}_{+}^{N}$ denotes the positive orthant $\left\{x \in \mathbb{R}^{N}: x \geq 0\right\}$ where we use the partial order for $x, y \in \mathbb{R}^{N}$ given by 
$x \geq y \Leftrightarrow x_{i} \geq y_{i}, i=1, \ldots, N$ and $x \geq y \Leftrightarrow \exists i: x_{i}<y_{i}$, $x>y \Leftrightarrow x_{i}>y_{i}, i=1, \ldots, N$.

We denote the Euclidean norm by $|\cdot|$. For $x=\left(x_{1}, \ldots, x_{N}\right)^{T}$ defined on an interval $I \subset \mathbb{R}$, we define $\|x\|_{\mathrm{I}}:=$ $\max _{i} \max _{t \in I}\left\{\left|x_{i}(t)\right|\right\}$.

$\nabla V$ denotes the gradient of a function $V$. The upper right-hand side derivative of a locally Lipschitz continuous function $V: \mathbb{R}^{N} \rightarrow \mathbb{R}_{+}$along $x(t) \in \mathbb{R}^{N}$ is defined by

$$
\mathrm{D}^{+} V(x(t))=\limsup _{h \rightarrow 0^{+}} \frac{V(x(t+h))-V(x(t))}{h} .
$$

Let $\theta \in \mathbb{R}_{+}$be the maximum involved delay. The function $x_{t}:[-\theta, 0] \rightarrow \mathbb{R}^{N}$ is given by $x_{t}(\tau):=x(t+\tau), \tau \in[-\theta, 0]$ and we define $\left\|x_{t}\right\|:=\max _{t-\theta \leq s \leq t}|x(s)|$. For $a, b \in \mathbb{R}$, $a<b$, let $C\left([a, b] ; \mathbb{R}^{N}\right)$ denote the Banach space of piecewise right-continuous functions defined on $[a, b]$ equipped with the norm $\|\cdot\|_{[a, b]}$ and take values in $\mathbb{R}^{N}$.

Definition 2.1. Classes of comparison functions are:

$\mathcal{K}:=\left\{\gamma: \mathbb{R}_{+} \rightarrow \mathbb{R}_{+} \mid \gamma\right.$ is continuous, $\gamma(0)=0$ and strictly increasing $\}$,

$\mathcal{K}_{\infty}:=\{\gamma \in \mathcal{K} \mid \gamma$ is unbounded $\}$,

$\mathcal{L}:=\left\{\gamma: \mathbb{R}_{+} \rightarrow \mathbb{R}_{+} \mid \gamma\right.$ is continuous and decreasing with $\left.\lim _{t \rightarrow \infty} \gamma(t)=0\right\}$,

$\mathcal{K} \mathcal{L}:=\left\{\beta: \mathbb{R}_{+} \times \mathbb{R}_{+} \rightarrow \mathbb{R}_{+} \mid \stackrel{t \rightarrow \infty}{\beta}\right.$ is continuous, $\beta(\cdot, t) \in \mathcal{K}, \beta(r, \cdot) \in \mathcal{L}, \forall t, r \geq 0\}$.

Note that for $\gamma \in \mathcal{K}_{\infty}$ the inverse function $\gamma^{-1}$ always exists and $\gamma^{-1} \in \mathcal{K}_{\infty}$.

\section{DELAY-FREE INTERCONNECTED IMPULSIVE SYSTEMS}

We consider an interconnection of $n$ impulsive subsystems with inputs

$$
\begin{aligned}
& \dot{x}_{i}(t)=f_{i}\left(x_{1}(t), \ldots, x_{n}(t), u_{i}(t)\right), t \neq t_{i \tilde{k}}, \\
& x_{i}(t)=g_{i}\left(x_{1}^{-}(t), \ldots, x_{n}^{-}(t), u_{i}^{-}(t)\right), t=t_{i \tilde{k}},
\end{aligned}
$$

$x_{i} \in \mathbb{R}^{N_{i}}, u_{i} \in \mathbb{R}^{M_{i}}$, where $\left\{t_{i 1}, t_{i 2}, t_{i 3}, \ldots\right\}$ is a strictly increasing sequence of impulsive times in $\left(t_{0}, \infty\right)$ for some initial time $t_{0}$ of the $i$ th subsystem. We assume further $t_{0}=0$. The set of impulsive times is assumed to be either finite or infinite and unbounded. Given a sequence $\left\{t_{i \tilde{k}}\right\}$ and a pair of times $s, t$ satisfying $t_{0} \leq s<t, N_{i}(t, s)$ denotes the number of impulsive times $t_{i \tilde{k}}$ in the semiopen interval $(s, t]$ of the $i$ th subsystem.

The state $x_{i}(t) \in \mathbb{R}^{N_{i}}$ of the $i$ th subsystem is absolutely continuous between impulses; $u_{i}(t) \in \mathbb{R}^{M_{i}}$ is a locally bounded, Lebesgue-measurable input. We assume that functions $f_{i}: \mathbb{R}^{N_{i}} \times \mathbb{R}^{M_{i}} \rightarrow \mathbb{R}^{N_{i}}$ and $g_{i}: \mathbb{R}^{N_{i}} \times \mathbb{R}^{M_{i}} \rightarrow \mathbb{R}^{N_{i}}$ are locally Lipschitz continuous. All signals (states $x_{i}$ and inputs $u_{i}$ ) are assumed to be right-continuous and to have left limits at all times and we denote $x_{i}^{-}(t):=\lim _{s}{ }_{t} x_{i}(s)$, $u_{i}^{-}(t):=\lim _{s \nearrow t} u_{i}(s)$.

If we define $N:=N_{1}+\ldots+N_{n}, M:=M_{1}+\ldots+$ $M_{n}, x:=\left(x_{1}^{T}, \ldots, x_{n}^{T}\right)^{T}, u:=\left(u_{1}^{T}, \ldots, u_{n}^{T}\right)^{T}$ and $f:=$ $\left(f_{1}^{T}, \ldots, f_{n}^{T}\right)^{T}$ and the impulsive time sequence of the whole system $\left\{t_{k}\right\}$ by $\left\{t_{k}\right\}:=\left\{t \mid t=t_{i \tilde{k}}, \tilde{k} \in \mathbb{N}\right\}$. Furthermore we define $I_{k}:=\left\{i \mid t_{k}=t_{i \tilde{k}}\right\}, \bar{I}_{k}:=\left.\left\{i \mid t_{k} \neq t_{i \tilde{k}}\right\}\right|_{I_{k}}$ and $N(t, s)$ denotes the number of impulsive times in the semi-open interval $(s, t]$ of the whole system. Then $\left.x\right|_{I_{k}}:=$ $\left(0, \ldots, g_{i_{1}}, \ldots, \ldots, g_{i_{p}}, \ldots, 0\right)^{T}$, where $i_{j} \in I_{k}, j=1, \ldots, p$ and $\left.x\right|_{\bar{I}_{k}}:=\left(0, \ldots, x_{i_{1}}^{-}, \ldots, \ldots, x_{i_{l}}^{-}, \ldots, 0\right)^{T}$, where $i_{j} \in$ $\bar{I}_{k}, j=1, \ldots, l$.

With these definitions the interconnected system (1) can be described as a system of the form

$$
\begin{aligned}
& \dot{x}(t)=f(x(t), u(t)), \quad t \neq t_{k}, \\
& x(t)=\left.x\right|_{I_{k}}+\left.x\right|_{\bar{I}_{k}}=: g\left(x^{-}(t), u^{-}(t)\right), t=t_{k},
\end{aligned}
$$

$k \in \mathbb{N}$. The ISS property of impulsive systems is defined as follows, see Hespanha et al. [2008].

Definition 3.1. Assume that a sequence $\left\{t_{k}\right\}$ is given. We call system (2) input-to-state stable (ISS) if there exist functions $\beta \in \mathcal{K} \mathcal{L}, \gamma \in \mathcal{K}_{\infty}$, such that for every initial condition $x(0)$ and every input $u$ the corresponding solution to (2) exists globally and satisfies

$$
|x(t)| \leq \max \left\{\beta(|x(0)|, t), \gamma\left(\|u\|_{[0, t]}\right)\right\}, \quad \forall t \geq 0 .
$$

The supremum norm of an input $u$ on the interval $\left[t_{0}, t\right]$ is redefined by

$$
\|u\|_{\left[t_{0}, t\right]}:=\max \left\{\operatorname{ess}_{s \in\left[t_{0}, t\right]}|u(s)|, \sup _{t_{k} \in\left[t_{0}, t\right]}\left|u\left(t_{k}\right)\right|\right\} .
$$

The impulsive system (2) is uniformly ISS over a given class $\mathcal{S}$ of admissible sequences of impulsive times if (3) holds for every sequence in $\mathcal{S}$, with functions $\beta$ and $\gamma$ that are independent of the choice of the sequence.

For a system with several inputs we use the following Definition 3.2. Assume that a sequence $\left\{t_{i \tilde{k}}\right\}$ is given. The $i$ th subsystem of (2) is ISS if there exist $\beta_{i} \in \mathcal{K} \mathcal{L}$, $\gamma_{i j}, \gamma_{i} \in \mathcal{K}_{\infty} \cup\{0\}$ such that for every initial condition $x_{i}(0)$ and every input $u_{i}$ the corresponding solution to (1) exists globally and satisfies for all $t \geq 0$

$\left|x_{i}(t)\right| \leq \max \left\{\beta_{i}\left(\left|x_{i}(0)\right|, t\right), \max _{j, j \neq i} \gamma_{i j}\left(\left\|x_{j}\right\|_{[0, t]}\right), \gamma_{i}\left(\|u\|_{[0, t]}\right)\right\}$

Functions $\gamma_{i j}$ are called gains. The impulsive system (1) is uniformly $I S S$ over a given class $\mathcal{S}$ of admissible sequences of impulsive times if (4) holds for every sequence in $\mathcal{S}$, with functions $\beta_{i}$ and $\gamma_{i}, \gamma_{i j}$ that are independent of the choice of the sequence.

For stability analysis of impulsive systems we use exponential Lyapunov functions, see Hespanha et al. [2008].

Definition 3.3. We say that a function $V: \mathbb{R}^{N} \rightarrow \mathbb{R}$ is an exponential ISS-Lyapunov function for (2) with rate coefficients $c, d \in \mathbb{R}$ if $V$ is locally Lipschitz, positive definite, radially unbounded, and satisfies

$$
V(x) \geq \gamma(|u|) \Rightarrow \nabla V(x) \cdot f(x, u) \leq-c V(x)
$$

for almost all $x$, all $u$ and

$$
V(x) \geq \gamma(|u|) \Rightarrow V(g(x, u)) \leq e^{-d} V(x)
$$

for all $x, u$, where $\gamma$ is some function from $\mathcal{K}_{\infty}$.

Condition (5) states, that if $c$ is positive then the function $V$ decreases. On the other hand, if $c<0$ then the function $V$ may increase. Condition (6) states, that if $d$ is positive then the jump (impulse) decreases the magnitude of $V$. On the other hand, if $d<0$ then the jump (impulse) may increase the magnitude of $V$.

Remark 3.4. Note that in Hespanha et al. [2008] the conditions (5) and (6) are in dissipative form. By Proposition 2.6 
in Cai and Teel [2009] the conditions in dissipative form are equivalent to the conditions in implication form, used in Definition 3.3, but coefficients $c, d$ may be different.

Similarly we define Lyapunov functions for subsystems. Definition 3.5. Function $V_{i}: \mathbb{R}^{N_{i}} \rightarrow \mathbb{R}$ is an exponential ISS-Lyapunov function for the $i$ th subsystem of (1) with rate coefficients $c_{i}, d_{i} \in \mathbb{R}$ if there exist functions $V_{j}, j=1, \ldots, n$, which are continuous, proper and positive definite and locally Lipschitz continuous on $\mathbb{R}^{N_{j}} \backslash\{0\}$ and $V_{i}$ satisfies

$$
\begin{gathered}
V_{i}\left(x_{i}\right) \geq \max \left\{\max _{j, j \neq i} \gamma_{i j}\left(V_{j}\left(x_{j}\right)\right), \gamma_{i}\left(\left|u_{i}\right|\right)\right\} \Rightarrow \\
\nabla V_{i}\left(x_{i}\right) \cdot f_{i}\left(x, u_{i}\right) \leq-c_{i} V_{i}\left(x_{i}\right)
\end{gathered}
$$

for almost all $x$, all $u_{i}$ and

$$
\begin{gathered}
V_{i}\left(x_{i}\right) \geq \max \left\{\max _{j, j \neq i} \gamma_{i j}\left(V_{j}\left(x_{j}\right)\right), \gamma_{i}\left(\left|u_{i}\right|\right)\right\} \Rightarrow \\
V_{i}\left(g_{i}\left(x, u_{i}\right)\right) \leq e^{-d_{i}} V_{i}\left(x_{i}\right)
\end{gathered}
$$

for all $x, u_{i}$, where $\gamma_{i j}, \gamma_{i}$ are some functions from $\mathcal{K}_{\infty}$.

The rate coefficients $c_{i}, d_{i}$ are not required to be nonnegative and therefore $V_{i}$ may not decrease even if $u=0$. Note that in general $\gamma, \gamma_{i j}, \gamma_{i}$ in the definitions of ISS and ISS-Lyapunov functions are different.

In Hespanha et al. [2008] the following theorem was proved which establishes stability of a single impulsive system even if one of rate coefficients is not positive.

Theorem 3.6. Let $V$ be an exponential ISS-Lyapunov function for $(2)$ with rate coefficients $c, d \in \mathbb{R}$ with $d \neq 0$. For arbitrary constants $\mu, \lambda>0$, let $\mathcal{S}[\mu, \lambda]$ denote the class of impulsive time sequences $\left\{t_{k}\right\}$ satisfying

$$
-d N(t, s)-(c-\lambda)(t-s) \leq \mu, \quad \forall t \geq s \geq 0 .
$$

Then the system (2) is uniformly ISS over $\mathcal{S}[\mu, \lambda]$.

When $d=0$ the theorem can be applied, because (9) holds for every $d<0$. This case and the case $c=0$ were investigated in more detail in Hespanha et al. [2008], Section 6 .

Condition (9) guarantees stability of the impulsive system even if the continuous or discontinuous behavior is unstable. For example, if the continuous behavior is unstable, which means $c<0$, then this condition assumes that the discontinuous behavior has to stabilize the system $(d>0)$ and the jumps have to occur often enough. Opposite if the discontinuous behavior is unstable $(d<0)$ and the continuous behavior is stable $(c>0)$ then the jumps have to occur rarely, which stabilizes the system.

In general even if all subsystems of (1) are ISS, the whole system (2) may be not ISS. Thus we are looking for the conditions that guarantee ISS of (2). We collect the nonlinear gains $\gamma_{i j}$ of the subsystems in a matrix $\Gamma=\left(\gamma_{i j}\right)_{n \times n}$, $i, j=1, \ldots, n$ denoting $\gamma_{i i} \equiv 0, i=1, \ldots, n$ for completeness, see Dashkovskiy et al. [2007], Rüffer [2007]. Note that this matrix describes in particular the interconnection topology of the whole network, moreover it contains the information about the mutual influence between the subsystems. We also introduce the gain operator $\Gamma: \mathbb{R}_{+}^{n} \rightarrow \mathbb{R}_{+}^{n}$ defined by

$$
\Gamma(s):=\left(\max _{j=1}^{n} \gamma_{1 j}\left(s_{j}\right), \ldots, \max _{j=1}^{n} \gamma_{n j}\left(s_{j}\right)\right), s \in \mathbb{R}_{+}^{n} .
$$

To show one of the main results we need the notion of a so called $\Omega$-path, see Dashkovskiy et al. [2009], Rüffer [2009]. A function $\sigma=\left(\sigma_{1}, \ldots, \sigma_{n}\right)^{T}: \mathbb{R}_{+}^{n} \rightarrow \mathbb{R}_{+}^{n}$, where $\sigma_{i} \in \mathcal{K}_{\infty}$ is called an $\Omega$-path, if it possesses the following properties:

(i) $\sigma_{i}^{-1}$ is locally Lipschitz continuous on $(0, \infty)$;

(ii) for every compact set $P \subset(0, \infty)$ there are finite constants $0<K_{1}<K_{2}$ such that for all points of differentiability of $\sigma_{i}^{-1}$ we have

$$
0<K_{1} \leq\left(\sigma_{i}^{-1}\right)^{\prime}(r) \leq K_{2}, \quad \forall r \in P
$$

$$
\Gamma(\sigma(r))<\sigma(r), \forall r>0 .
$$

The next theorem provides a condition for the existence of an $\Omega$-path.

Theorem 3.7. Let $\Gamma \in\left(\mathcal{K}_{\infty} \cup\{0\}\right)^{n \times n}$ be a gain matrix. If $\Gamma$ satisfies the small-gain condition

$$
\Gamma(s) \nsupseteq s, \forall s \in \mathbb{R}_{+}^{n} \backslash\{0\},
$$

then there exists an $\Omega$-path $\sigma$ with respect to $\Gamma$. This path can be chosen piecewise linear.

The proof can be found in Dashkovskiy et al. [2009], Theorem 5.2, see also Rüffer [2009], however only the existence is proved in these works.

Now we can formulate one of the main results that is an ISS small-gain theorem for impulsive systems without timedelays. This theorem allows to construct an ISS Lyapunov function for the whole interconnection.

\subsection{Main result}

Theorem 3.8. Assume that each subsystem of (1) has an exponential ISS-Lyapunov function $V_{i}$ with corresponding ISS-Lyapunov gains $\gamma_{i j}$ and rate coefficients $c_{i}, d_{i}, d_{i} \neq$ 0 . Define $c:=\min _{i}$ and $d:=\min _{i} d_{i}$. For arbitrary constants $\mu, \lambda>0$, let $\mathcal{S}[\mu, \lambda]$ denote the class of impulsive time sequences $\left\{t_{k}\right\}$ of the whole system. If the following holds

i) $\mathcal{S}[\mu, \lambda]$ satisfies condition (9),

ii) $\Gamma=\left(\gamma_{i j}\right)_{n \times n}$ satisfies the small-gain condition (12),

then the impulsive system (2) is uniformly ISS over $\mathcal{S}[\mu, \lambda]$ and the exponential ISS-Lyapunov function is given by

$$
V(x):=\max _{i}\left\{\sigma_{i}^{-1}\left(V_{i}\left(x_{i}\right)\right)\right\},
$$

where $\sigma=\left(\sigma_{1}, \ldots, \sigma_{n}\right)^{T}$ is a piecewise linear $\Omega$-path. The gain is given by $\gamma(r):=\max _{i} \sigma_{i}^{-1}\left(\gamma_{i}(r)\right)$.

The small-gain condition ii) is used for example in Dashkovskiy et al. [2007, 2009] to verify the ISS property of interconnected systems. The condition i) is the average dwell-time condition for interconnections of impulsive systems. For single impulsive systems, which means there is only one $c$ and one $d$ this condition can be found in Hespanha et al. [2008].

Proof. As the small gain condition (12) is satisfied it follows from Theorem 3.7 that there exists an $\Omega$-path $\sigma$ with respect to $\Gamma$. We can choose this path to be piecewise linear. Let us define

$$
V(x)=\max _{i} \sigma_{i}^{-1}\left(V_{i}\left(x_{i}\right)\right)
$$


and show that this function is an exponential ISSLyapunov function for the system (2). It can be easily checked that this function is locally Lipschitz, positive definite and radially unbounded.

For any $i \in\{1, \ldots, n\}$ consider open domains

$M_{i} \in \mathbb{R}^{N} \backslash\{0\}$ defined by

$$
\begin{aligned}
M_{i}:=\{ & \left(x_{1}^{T}, \ldots, x_{n}^{T}\right)^{T} \in \mathbb{R}^{N} \backslash\{0\}: \\
& \left.\sigma_{i}^{-1}\left(V_{i}\left(x_{i}\right)\right)>\max _{j \neq i}\left\{\sigma_{j}^{-1}\left(V_{j}\left(x_{j}\right)\right)\right\}\right\} .
\end{aligned}
$$

Now for any $\hat{x}=\left(\hat{x}_{1}^{T}, \ldots, \hat{x}_{n}^{T}\right)^{T} \in \mathbb{R}^{N} \backslash\{0\}$ there is at least one $i \in\{1, \ldots, n\}$ such that $\hat{x} \in M_{i}$ and it follows, that there is a neighborhood $U$ of $\hat{x}$ such that $V(x)=$ $\sigma_{i}^{-1}\left(V_{i}\left(x_{i}\right)\right)$ holds for all $x \in U$.

We define $\gamma(r):=\max _{i} \sigma_{i}^{-1}\left(\gamma_{i}(r)\right), r>0$ and assume $V(x) \geq \gamma(|u|)$. It follows from (11) that

$$
\begin{aligned}
V_{i}\left(x_{i}\right) & =\sigma_{i}(V(x)) \geq \max \left\{\max _{j}\left(\gamma_{i j}\left(\sigma_{j}(V(x))\right)\right), \sigma_{i}(\gamma(|u|))\right\} \\
& \geq \max \left\{\max _{j} \gamma_{i j}\left(V_{j}(x)\right), \gamma_{i}\left(\left|u_{i}\right|\right)\right\} .
\end{aligned}
$$

Let $\left[s_{l}, s_{l+1}\right], s_{l}<s_{l+1}, l=0,1, \ldots$ be an interval where $\sigma_{i}$ is linear, i.e., $\sigma_{i}(t)=a_{i l} s, s \in\left[s_{l}, s_{l+1}\right]$. Then for all intervals with $\sigma_{i}(s)=a_{i l} s$ and from (7) we obtain for almost all $x$ and $V_{i}\left(x_{i}\right) \in\left[s_{l}, s_{l+1}\right]$

$$
\begin{aligned}
\dot{V}(x) & =\left(\sigma_{i}^{-1}\right)^{\prime}\left(V_{i}\left(x_{i}\right)\right) \nabla V_{i}\left(x_{i}\right) \cdot f_{i}\left(x, u_{i}\right) \\
& \leq-\frac{1}{a_{i l}} c_{i} V_{i}\left(x_{i}\right)=-c_{i} V(x) .
\end{aligned}
$$

By the definition of $c:=\min _{i} c_{i}$ the function $V$ satisfies (5). As $d:=\min _{i} d_{i}$ it holds

$$
\begin{aligned}
V(g(x, u)) & =\frac{1}{a_{i l}}\left(V_{i}\left(g_{i}\left(x_{1}, \ldots, x_{n}, u_{i}\right)\right)\right) \\
& \leq \frac{1}{a_{i l}} e^{-d_{i}} V_{i}\left(x_{i}\right) \leq e^{-d} V(x), V_{i}\left(x_{i}\right) \in\left[s_{l}, s_{l+1}\right],
\end{aligned}
$$

i.e., $V$ satisfies condition (6).

All conditions of Definition 3.3 are satisfied and thus $V$ is the exponential ISS-Lyapunov function of system (2). By assumption i) there exist $\mu, \lambda>0$ such that $-d N(t, s)-$ $c(t-s) \leq \mu-\lambda(t-s), \forall t \geq s \geq 0$ we can apply Theorem 3.6 and the overall system is uniformly ISS over $S[\mu, \lambda]$.

Using $c=\min _{i} c_{i}, d=\min _{i} d_{i}$ in the average dwelltime condition some kind of conservativeness may occur, which means we cannot verify the ISS property for an interconnected impulsive system by the application of Theorem 3.8, although the system possesses the ISS property. Remark 3.9. If both dynamics, the continuous and the discontinuous, are stable, which means that $c, d>0$, then the condition (9) in Theorem 3.8 is not necessary, according to Theorem 2 in Hespanha et al. [2008].

The following section provides a similar small-gain result, but for impulsive systems with time-delays.

\section{INTERCONNECTED IMPULSIVE SYSTEMS WITH TIME-DELAYS}

Consider $n$ interconnected impulsive systems with timedelays of the form

$$
\begin{aligned}
& \dot{x}_{i}(t)=f_{i}\left(x_{1}^{t}, \ldots, x_{n}^{t}, u_{i}(t)\right), t \neq t_{i \tilde{k}}, \\
& x_{i}(t)=g_{i}\left(\left(x_{1}^{t}\right)^{-}, \ldots,\left(x_{n}^{t}\right)^{-}, u_{i}^{-}(t)\right), t=t_{i \tilde{k}},
\end{aligned}
$$

$\tilde{k} \in \mathbb{N}, i=1, \ldots, n$, where the same assumptions on the system as in the delay-free case are considered with the following differences: We denote $x_{i}^{t}(\tau):=x_{i}(t+$ $\tau), \tau \in[-\theta, 0]$, where $\theta$ is the maximum involved delay and $\left(x_{i}^{t}\right)^{-}(\tau):=x_{i}^{-}(t+\tau):=\lim _{s}{ }_{t} x_{i}(s+\tau), \tau \in[-\theta, 0]$. We assume that the functionals $f_{i}: C\left([-\theta, 0], \mathbb{R}^{N_{1}}\right) \times \ldots \times$ $C\left([-\theta, 0], \mathbb{R}^{N_{n}}\right) \times \mathbb{R}^{M_{i}} \rightarrow \mathbb{R}^{N_{i}}$ and $g_{i}: C\left([-\theta, 0], \mathbb{R}^{N_{1}}\right) \times$ $\ldots \times C\left([-\theta, 0], \mathbb{R}^{N_{n}}\right) \times \mathbb{R}^{M_{i}} \rightarrow \mathbb{R}^{N_{i}}$ are locally Lipschitz continuous.

If we define $t_{k}, N, M, x, u, f$ and $g$ as in the delay-free case, then (16) becomes the system of the form

$$
\begin{aligned}
& \dot{x}(t)=f\left(x_{t}, u(t)\right), t \neq t_{k}, k \in \mathbb{N}, \\
& x(t)=g\left(x_{t}^{-}, u^{-}(t)\right), t=t_{k}, k \in \mathbb{N} .
\end{aligned}
$$

We assume that the regularity conditions in Ballinger and Liu [1999] for the existence and uniqueness of solutions of systems (16) and (17) are satisfied. For any $\xi_{i} \in$ $C\left([-\theta, 0], \mathbb{R}^{N_{i}}\right)$ the solution of the $i$ th subsystem of $(16)$ is denoted by $x_{i}\left(t, t_{0}, \xi_{i}\right)$ or $x_{i}(t)$ for short, satisfying the initial condition $x_{i}^{t_{0}}(\tau)=\xi_{i}(\tau), \tau \in[-\theta, 0]$. In a similar way we denote the solution of the system (17) by $x\left(t, t_{0}, \xi\right)$ or $x(t)$ for short for any $\xi \in C\left([-\theta, 0], \mathbb{R}^{N}\right)$ that exists in a maximal interval $[-\theta, b), 0<b \leq+\infty$, satisfying the initial condition $x_{t_{0}}(\tau)=\xi(\tau), \tau \in[-\theta, 0]$.

The definition of ISS is then similar to the delay-free case: Definition 4.1. Suppose that a sequence $\left\{t_{k}\right\}$ is given. We call system (17) ISS if there exist functions $\beta \in \mathcal{K} \mathcal{L}$, $\gamma \in \mathcal{K}_{\infty}$, such that for every initial condition $x_{0}=\xi \in$ $C([-\theta, 0])$ and every input $u$ the corresponding solution to $(17)$ exists globally and satisfies

$$
|x(t)| \leq \max \left\{\beta\left(\|\xi\|_{[-\theta, 0]}, t\right), \gamma\left(\|u\|_{[0, t]}\right)\right\}, \quad \forall t \geq 0 .
$$

The impulsive system (17) is uniformly ISS over a given class $\mathcal{S}$ of admissible sequences of impulsive times if (18) holds for every sequence in $\mathcal{S}$, with functions $\beta$ and $\gamma$ that are independent of the choice of the sequence.

For the stability analysis of systems of the form (17) Razumikhin-type theorems were proved in Chen and Zheng [2009]. In our second main result we use the definition of an exponential ISS-Lyapunov-Razumikhin function in implication form and the (reverse) average dwell-time condition from Hespanha et al. [2008].

Definition 4.2. A locally Lipschitz continuous function $V: \mathbb{R}^{N} \rightarrow \mathbb{R}_{+}$is called an exponential ISS-LyapunovRazumikhin function for system (17), if there exist $\psi_{1}, \psi_{2} \in \mathcal{K}_{\infty}, \gamma_{u} \in \mathcal{K}, \gamma_{d} \in \mathcal{K}_{\infty}$ and scalars $c, d \in \mathbb{R}$ such that

$$
\begin{gathered}
\psi_{1}(|x|) \leq V(x) \leq \psi_{2}(|x|), \\
V(x) \geq \max \left\{\gamma_{d}\left(|| V_{d}(x)||\right), \gamma_{u}(|u(t)|)\right\} \\
\Rightarrow \mathrm{D}^{+} V(x) \leq-c V(x), \\
V(x) \geq \max \left\{\gamma_{d}\left(|| V_{d}(x)||\right), \gamma_{u}(|u(t)|)\right\} \\
\Rightarrow V(g(x, u)) \leq e^{-d} V(x),
\end{gathered}
$$

hold for all $x(t) \in \mathbb{R}^{N}$ and $u(t) \in \mathbb{R}^{M}$, where $V_{d}(x(t)):=$ $V(x(t+\tau)), \tau \in[-\theta, 0]$.

Remark 4.3. Note that in Chen and Zheng [2009] the conditions (20) and (21) are in dissipative form. By Proposition 2.6 in Cai and Teel [2009] the conditions in dissipa- 
tive form are equivalent to the conditions in implication form, used in Definition 4.2, where the coefficients $c, d$ are different in general.

The following proposition is similar to Theorem 1 in Hespanha et al. [2008]. Here we adopt the approach used in this paper to time-delay systems. In Chen and Zheng [2009] a different approach for the characterization of the condition on the time intervals of the impulses is used, but the statement is the same. By combining the results in the two previous mentioned papers we can state the following: Proposition 4.4. Let $V$ be an exponential ISS-LyapunovRazumikhin function for (17) with $c, d \in \mathbb{R}$. For arbitrary constants $\mu, \lambda \in \mathbb{R}_{+}$, let $\mathcal{S}[\mu, \lambda]$ denote the class of impulse time sequences $\left\{t_{k}\right\}$ satisfying (9). If $\gamma_{d}<\mathrm{Id}$, then the system (17) is uniformly ISS over $\mathcal{S}[\mu, \lambda]$.

The proof is skipped, because it is a combination of the proofs of Theorem 1 in Hespanha et al. [2008] and Theorems 1 and 2 in Chen and Zheng [2009] with an exponential ISS-Lyapunov-Razumikhin function as in Definition 4.2.

Now we consider interconnected impulsive time-delay systems and define the ISS property and Lyapunov functions of the subsystems as follows:

Definition 4.5. Suppose that a sequence $\left\{t_{i \tilde{k}}\right\}$ is given. The $i$ th subsystem of (16) is $I S S$ if there exist $\beta_{i} \in \mathcal{K} \mathcal{L}$, $\gamma_{i j}, \gamma_{i}^{u} \in \mathcal{K}_{\infty} \cup\{0\}$ such that for every initial condition $x_{i}^{0}=\xi_{i}$ and every input $u_{i}$ the corresponding solution to the $i$ th subsystem of (16) exists globally and satisfies

$$
\left|x_{i}(t)\right| \leq \max \left\{\beta_{i}\left(\left\|\xi_{i}\right\|_{[-\theta, 0]}, t\right),\right.
$$

for all $t \geq 0$. The $i$ th subsystem of (16) is uniformly ISS over a given class $\mathcal{S}$ of admissible sequences of impulsive times if $(22)$ holds for every sequence in $\mathcal{S}$, with functions $\beta_{i}, \gamma_{i j}$ and $\gamma_{i}^{u}$ that are independent of the choice of the sequence.

Definition 4.6. A locally Lipschitz continuous function $V_{i}: \mathbb{R}^{N_{i}} \rightarrow \mathbb{R}_{+}$is called an exponential ISS-LyapunovRazumikhin function of the ith subsystem of (16), if there exist functions $V_{j}, j=1, \ldots, n$, which are continuous, proper and positive definite and locally Lipschitz continuous on $\mathbb{R}^{N_{j}} \backslash\{0\}$ and there exist $\gamma_{i}^{u} \in \mathcal{K} \cup\{0\}, \gamma_{i j} \in \mathcal{K}_{\infty} \cup$ $\{0\}, j=1, \ldots, n$ and scalars $c_{i}, d_{i} \in \mathbb{R}$, such that

$$
\begin{gathered}
\quad V_{i}\left(x_{i}\right) \geq \max \left\{\max _{j} \gamma_{i j}\left(\left\|V_{j}^{d}\left(x_{j}(t)\right)\right\|\right), \gamma_{i}^{u}\left(\left|u_{i}(t)\right|\right)\right\} \\
\Rightarrow \quad \mathrm{D}^{+} V_{i}\left(x_{i}\right) \leq-c_{i} V_{i}\left(x_{i}\right), \\
V_{i}\left(x_{i}\right) \geq \max \left\{\max _{j} \gamma_{i j}\left(\left\|V_{j}^{d}\left(x_{j}(t)\right)\right\|\right), \gamma_{i}^{u}\left(\left|u_{i}(t)\right|\right)\right\} \\
\Rightarrow \quad V_{i}\left(g_{i}\left(x_{1}, \ldots, x_{n}, u_{i}\right)\right) \leq e^{-d_{i}} V_{i}\left(x_{i}\right),
\end{gathered}
$$

hold for all $x=\left(x_{1}^{T}, \ldots, x_{n}^{T}\right)^{T} \in \mathbb{R}^{N}$ and $u_{i} \in \mathbb{R}^{M_{i}}$, where $V_{j}^{d}\left(x_{j}(t)\right):=V_{j}\left(x_{j}(t+\tau)\right), \tau \in[-\theta, 0]$. Furthermore we define the gain-matrix $\Gamma:=\left(\gamma_{i j}\right)_{n \times n}$ and the map $\Gamma: \mathbb{R}_{+}^{n} \rightarrow$ $\mathbb{R}_{+}^{n}$ by $\Gamma(s):=\left(\max _{j} \gamma_{1 j}\left(s_{j}\right), \ldots, \max _{j} \gamma_{n j}\left(s_{j}\right)\right)^{T}, s \in \mathbb{R}_{+}^{n}$.

Now we state our second main result: the ISS small-gain theorem for interconnected impulsive systems with timedelays. We construct the Lyapunov-Razumikhin function and the gain of the overall system under a small-gain condition. The necessary ADT condition on the size of the time intervals between impulses is the same as in the delay-free case.

\subsection{Main result}

Theorem 4.7. Assume each subsystem of (16) has an exponential ISS-Lyapunov-Razumikhin function with $c_{i}, d_{i} \in$ $\mathbb{R}, d_{i} \neq 0$ and gains $\gamma_{i j}$. Define $c:=\min _{i} c_{i}$ and $d:=$ $\min _{i} d_{i}$. For arbitrary constants $\mu, \lambda>0$, let $\mathcal{S}[\mu, \lambda]$ denote the class of impulsive time sequences $\left\{t_{k}\right\}$ of the whole system. If the following holds

i) $\mathcal{S}[\mu, \lambda]$ satisfies condition (9),

ii) $\Gamma=\left(\gamma_{i j}\right)_{n \times n}$ satisfies the small-gain condition (12),

then the whole system (17) is uniformly ISS over $\mathcal{S}[\mu, \lambda]$ and the exponential ISS-Lyapunov-Razumikhin function is given by $V(x):=\max _{i}\left\{\sigma_{i}^{-1}\left(V_{i}\left(x_{i}\right)\right)\right\}$, where $\sigma=$ $\left(\sigma_{1}, \ldots, \sigma_{n}\right)^{T}$ is a piecewise linear $\Omega$-path. The gains are given by $\gamma_{d}(r) \quad:=\max _{k, j} \sigma_{k}^{-1}\left(\gamma_{k j}\left(\sigma_{j}(r)\right)\right)$, $\gamma_{u}(r):=\max _{i} \sigma_{i}^{-1}\left(\gamma_{i}^{u}(r)\right)$.

The proof goes along the line of the proof of Theorem 3.8 with according changes to time-delay systems.

Proof. Let $0 \neq x=\left(x_{1}^{T}, \ldots, x_{n}^{T}\right)^{T}$. We define

$$
V(x):=\max _{i}\left\{\sigma_{i}^{-1}\left(V_{i}\left(x_{i}\right)\right)\right\}
$$

and show that $V$ is the exponential ISS-LyapunovRazumikhin function for the overall system. $V$ satisfies (19), which can be easily checked. Note that $V$ is locally Lipschitz continuous. For any $i \in\{1, \ldots, n\}$ consider open domains $M_{i} \in \mathbb{R}^{N} \backslash\{0\}$ defined as in (15). Now for any $\hat{x}=\left(\hat{x}_{1}^{T}, \ldots, \hat{x}_{n}^{T}\right)^{T} \in \mathbb{R}^{N} \backslash\{0\}$ there is at least one $i \in\{1, \ldots, n\}$ such that $\hat{x} \in M_{i}$ and it follows, that there is a neighborhood $U$ of $\hat{x}$ such that $V(x)=\sigma_{i}^{-1}\left(V_{i}\left(x_{i}\right)\right)$ holds for all $x \in U$.

We define the gains $\gamma_{d}(r):=\max _{k, j} \sigma_{k}^{-1}\left(\gamma_{k j}\left(\sigma_{j}(r)\right)\right)$, $\gamma_{u}(r):=\max _{i} \sigma_{i}^{-1}\left(\gamma_{i}^{u}(r)\right), r>0$ and assume

$$
V(x) \geq \max \left\{\gamma_{d}\left(|| V_{d}(x)||\right), \gamma_{u}(|u|)\right\} .
$$

Note that $\gamma_{d}(r)<r$, by (11). It follows

$$
\begin{gathered}
V_{i}\left(x_{i}\right) \geq \sigma_{i}\left(\operatorname { m a x } \left\{\max _{k j} \sigma_{k}^{-1}\left(\gamma_{k j}\left(\sigma_{j}\left(|| V_{d}(x)||\right)\right)\right),\right.\right. \\
\left.\left.\max _{i} \sigma_{i}^{-1}\left(\gamma_{i}^{u}(|u|)\right)\right\}\right) \\
\geq \max \left\{\max _{j} \gamma_{i j}\left(|| V_{j}^{d}(x)||\right), \gamma_{i}^{u}\left(\left|u_{i}\right|\right)\right\} .
\end{gathered}
$$

Let $\left[s_{l}, s_{l+1}\right], s_{l}<s_{l+1}, l=0,1, \ldots$ be an interval where $\sigma_{i}$ is linear, i.e., $\sigma_{i}(s)=a_{i l} s, s \in\left[s_{l}, s_{l+1}\right]$. Then for all intervals with $\sigma_{i}(s)=a_{i l} s$ and from (23) we obtain

$$
\mathrm{D}^{+} V(x)=\mathrm{D}^{+} \frac{1}{a_{i l}} V_{i}\left(x_{i}\right) \leq-\frac{1}{a_{i l}} c_{i} V_{i}\left(x_{i}\right)=-c_{i} V(x),
$$

for almost all $x$ and for all $V_{i}\left(x_{i}\right) \in\left[s_{l}, s_{l+1}\right]$. By definition of $c:=\min _{i} c_{i}$ the function $V$ satisfies (20). By definition of $d:=\min _{i} d_{i}$ it holds

$$
V(g(x, u)) \leq \frac{1}{a_{i l}} e^{-d_{i}} V_{i}\left(x_{i}\right) \leq e^{-d} V(x), V_{i} \in\left[s_{l}, s_{l+1}\right]
$$

i.e., $V$ satisfies condition (21).

All conditions of Definition 4.2 are satisfied and $V$ is the exponential ISS-Lyapunov-Razumikhin function of the 
whole system of the form (17). Using that there exist $\mu, \lambda>0$ such that $-d N(t, s)-c(t-s) \leq \mu-\lambda(t-$ $s), \forall t \geq s \geq 0$ we can apply Proposition 4.4 and the whole system is uniformly ISS over $\mathcal{S}[\mu, \lambda]$.

Remark 4.8. The meaning of $c, d \in \mathbb{R}$ is the same as in the delay-free case. If both dynamics, the continuous and the discontinuous, are stable, which means that $c, d>0$, then the condition (9) in Theorem 4.7 is not necessary, according to Theorem 2 in Hespanha et al. [2008].

\section{CONCLUSIONS}

We have established conditions that guarantee input-tostate stability of a network of several impulsive systems with and without time-delays. These conditions are based on the interconnection structure of the network (smallgain condition) and on the sizes of time intervals between the impulses (average dwell-time condition). Our approach provides a method of construction of Lyapunov and Lyapunov-Razumikhin functions for the overall system.

\section{REFERENCES}

George Ballinger and Xinzhi Liu. Existence and uniqueness results for impulsive delay differential equations. Dynam. Contin. Discrete Impuls. Systems, 5(1-4):579591, 1999. Differential equations and dynamical systems (Waterloo, ON, 1997).

C. Cai and Andrew R. Teel. Characterizations of inputto-state stability for hybrid systems. Systems Control Lett., 58:47-53, 2009.

Wu-Hua Chen and Wei Xing Zheng. Brief paper: Inputto-state stability and integral input-to-state stability of nonlinear impulsive systems with delays. Automatica (Journal of IFAC), 45(6):1481-1488, 2009.

Sergey Dashkovskiy and Michael Kosmykov. Stability of networks of hybrid ISS systems. In Proceedings of the 48th IEEE Conference on Decision and Control, Shanghai, China, Dec. 16-18, 2009, pages 3870-3875, 2009.

Sergey Dashkovskiy and Lars Naujok. ISDS small-gain theorem and construction of ISDS Lyapunov functions for interconnected systems. Accepted in Systems and Control Letters, 2010. DOI:10.1016/j.sysconle.2010.03.004.

Sergey Dashkovskiy, Björn S. Rüffer, and Fabian R. Wirth. An ISS small gain theorem for general networks. Math. Control Signals Systems, 19(2):93-122, 2007.

Sergey Dashkovskiy, Björn S. Rüffer, and Fabian R. Wirth. Small gain theorems for large scale systems and construction of ISS Lyapunov functions, 2009. Accepted in SIAM Journal on Control and Optimization, available electronically: http://arxiv.org/pdf/0901.1842.

Sergey N. Dashkovskiy and Björn S. Rüffer. Local ISS of large-scale interconnections and estimates for stability regions. Systems \& Control Letters, 59(3-4):241 - 247, 2010.

Lars Grüne. Input-to-state dynamical stability and its Lyapunov function characterization. IEEE Trans. Automat. Control, 47(9):1499-1504, 2002.

Wassim M. Haddad, Vijay Sekhar Chellaboina, and Sergey G. Nersesov. Impulsive and hybrid dynamical systems. Princeton Series in Applied Mathematics. Princeton University Press, Princeton, NJ, 2006.

João P. Hespanha, Daniel Liberzon, and Andrew R. Teel. Lyapunov conditions for input-to-state stability of impulsive systems. Automatica J. IFAC, 44(11):2735-2744, 2008.

Hiroshi Ito. State-dependent scaling problems and stability of interconnected iISS and ISS systems. IEEE Transactions on Automatic Control, 51(10):1626-1643, 2006.

Hiroshi Ito, Sergey Dashkovskiy, and Fabian Wirth. On a small gain theorem for networks of iISS systems. In Proceedings of the 48th IEEE Conference on Decision and Control, Shanghai, China, Dec. 16-18, 2009, pages 4210-4215, 2009.

Z.-P. Jiang, A. R. Teel, and L. Praly. Small-gain theorem for ISS systems and applications. Math. Control Signals Systems, 7(2):95-120, 1994.

Zhong-Ping Jiang, Iven M. Y. Mareels, and Yuan Wang. A Lyapunov formulation of the nonlinear small-gain theorem for interconnected ISS systems. Automatica J. IFAC, 32(8):1211-1215, 1996.

Iasson Karafyllis and Zhong-Ping Jiang. A vector smallgain theorem for general nonlinear control systems, 2009. http://arxiv.org/pdf/0904.0755.

D. Nesic and A.R. Teel. A Lyapunov-based small-gain theorem for hybrid ISS systems. In Proceedings of the 47th IEEE Conference on Decision and Control, 2008, Cancun, Mexico, Dec. 9-11, 2008, pages 3380-3385, 2008.

P. Pepe and Z.-P. Jiang. A Lyapunov-Krasovskii methodology for ISS and iISS of time-delay systems. Systems Control Lett., 55(12):1006-1014, 2006.

B. S. Rüffer. Monotone inequalities, dynamical systems, and paths in the positive orthant of Euclidean $n$-space. Positivity, 2009. To appear, DOI:10.1007/s11117-0090016-5.

Björn Rüffer. Monotone dynamical systems, graphs, and stability of large-scale interconnected systems. $\mathrm{PhD}$ thesis, Fachbereich 3 (Mathematik \& Informatik) der Universität Bremen, 2007.

Robert Shorten, Fabian Wirth, Oliver Mason, Kai Wulff, and Christopher King. Stability criteria for switched and hybrid systems. SIAM Rev., 49(4):545-592, 2007.

Eduardo D. Sontag. Smooth stabilization implies coprime factorization. IEEE Trans. Automat. Control, 34(4): 435-443, 1989.

Eduardo D. Sontag. Comments on integral variants of ISS. Systems Control Lett., 34(1-2):93-100, 1998.

Eduardo D. Sontag and Yuan Wang. On characterizations of the input-to-state stability property. Systems Control Lett., 24(5):351-359, 1995.

Eduardo D. Sontag and Yuan Wang. New characterizations of input-to-state stability. IEEE Trans. Automat. Control, 41(9):1283-1294, 1996.

Andrew R. Teel. Connections between Razumikhin-type theorems and the ISS nonlinear small gain theorem. IEEE Trans. Automat. Control, 43(7):960-964, 1998.

Arjan van der Schaft and Hans Schumacher. An introduction to hybrid dynamical systems, volume 251 of Lecture Notes in Control and Information Sciences. SpringerVerlag London Ltd., London, 2000. 